\title{
MASS MOTIONS ASSOCIATED WITH SOLAR FLARES
}

\author{
SARA F. MARTIN \\ Big Bear Solar Observatory, California Institute of Technology, Pasadena, CA 91125, U.S.A.
}

\begin{abstract}
Mass motions are a principal means by which components of solar flares can be distinguished. Typical patterns of mass motions in $\mathbf{H} \alpha$ are described for chromospheric flare ribbons, remote chromospheric flare patches, flare loops, flaring arches, surges, erupting filaments and some expanding coronal features. Interrelationships between these phenomena are discussed and illustrations of each are presented.
\end{abstract}

\section{Introduction}

Throughout this review, solar flares are discussed as consisting of a number of components. As listed in Table I, these include loops, ribbons, arches, remote patches, surges, erupting filaments, and other expanding coronal features. Mass motions play an integral role in the existence of these components. It is the distinctly different velocity and spatial patterns of the mass motions of these flare components that has lead investigators to put them into separate categories and to assign them different names.

In Table I, the most closely related flare components in the chromosphere and corona are shown on the same line but in separate columns. The term 'coronal' in this paper refers to the volume of solar atmosphere above the chromosphere rather than to the typical temperature regime of that atmosphere.

TABLE I

\begin{tabular}{ll}
\hline Coronal flare components & Chromospheric flare components \\
\hline Flare loops & Ribbons \\
Flaring arches & Remote patches \\
Surges & \\
Erupting filaments & \\
Expanding coronal features & \\
\hline
\end{tabular}

The majority of flares have at least the two components listed in the first row in Table I, flare loops and chromospheric ribbons at the footpoints of the flare loops. Both are found in a wide variety of sizes and degrees of compactness. In $\mathrm{H} \alpha$ the ribbons are seen many minutes before the appearance of flare loops if and when conditions render the loops visible in $\mathrm{H} \alpha$. However, it is now well known that the loops are always present in $\mathrm{X}$-rays whenever the ribbons are seen in $\mathrm{H} \alpha$. The other phenomena apparently require specific additional conditions in order to occur in association with the loops and ribbons.

The literature on the mass motions involved in these many flare components is too extensive for an in-depth discussion on each one in the space allotted for this review. This presentation is limited to two specific objectives. The first goal is to summarize the general character of the mass motions of each of the above flare components by referring 
only to a few key papers and to previously unpublished, representative examples of each. The selection of key papers on a topic usually includes the first known papers, comprehensive papers, review papers and very recent papers, especially those which include references to earlier work. The second goal is to discuss and illustrate some of the relationships of these phenomena to each other as understood to date.

The illustrations in this paper are primarily $\mathrm{H} \alpha$ filtergrams. In depicting mass motions, $\mathrm{H} \alpha$ observations offer three advantages: (1) superior sharpness of detail and contrast (compared to current observations at most other wavelengths) afforded by the width and depth of the $\mathrm{H} \alpha$ line as observed with narrow band birefringent filters of $\frac{1}{4}$ or $\frac{1}{2} \AA$ passband, (2) a wide selection of events from an abundance of time-lapse observations taken in $\mathrm{H} \alpha$ over the last 3 solar cycles, and (3) a means of identifying the coronal flare components seen in projection against the chromospheric background.

The flare components that lie in the corona have parts or phases that include both very high temperatures (invisible in $\mathrm{H} \alpha$ ) and relatively low temperatures (visible in $\mathrm{H} \alpha$ ). The requirements for observing $\mathrm{H} \alpha$ flare components in the corona but against the chromospheric background is the existence of a plasma with a density of at least $10^{10}$ particles $\mathrm{cm}^{-3}$ and a temperature in the range of about $6-15 \times 10^{3} \mathrm{~K}$. A great many of the flare-associated mass events pass through this physical regime sometime during their evolution. Hence, very few flare components have been discovered in the UV or in X-rays whose existence was not already at least partially known or deduced from $\mathrm{H} \alpha$ observations. For these reasons, $\mathrm{H} \alpha$ is a good starting point for discussing mass motions. This review does not attempt to comprehensively discuss mass motions outside of the visible part of the spectrum. The author hopes this approach of using $\mathrm{H} \alpha$ observations will lay the groundwork for a series of more extensive reviews on each of the particular types of mass motions and their signatures as detected throughout the whole spectrum from $\mathrm{X}$-rays to radio wavelengths.

\section{Mass Motions within Chromospheric Flare Components}

\subsection{Chromospheric RIBBons}

The ribbons are distinguishable from the other forms of flare emission because they form simultaneously in the chromosphere on either side of a polarity inversion line. A polarity inversion line can be identified directly in a magnetogram displaying only the line-of-sight component of the chromospheric or photospheric magnetic field. Alternatively, many polarity inversion lines can be identified indirectly by the presence of a filament in filtergrams or spectroheliograms taken in chromospheric lines. The chromospheric ribbons are not usually symmetrically positioned on either side of a polarity inversion line. Also the ribbon patterns can be complex when several polarity inversion lines are involved in a single flare. This means that there can be multiple associated loop systems.

During most of their lifetimes, the flaring elements within the chromospheric flare ribbons have very small Doppler shifts on the order of $0-0.2 \AA$, corresponding to motions up to about $10 \mathrm{~km} \mathrm{~s}^{-1}$. However, the Doppler shifts of very bright elements, 
within the flare ribbons, known as 'flare kernals', can be as high as 1-2 $\AA$ for very intense flares. During their development, chromospheric flare elements are almost always Doppler-shifted or asymmetric towards the red (Ellison, 1952; Švestka, Kopecký, and Blaha, 1962; Tang, 1983; Ichimoto and Kurokawa, 1984).

In this paper, I follow some of the above authors in making a distinction between red-asymmetric profiles discovered by Waldmeier (1941) and red-shifted flare profiles later recognized by Teske (1962). According to Ellison (1952), who first used the term 'red asymmetry', the peak intensity is at line center and the red wing is brighter and more extensive than the blue wing. In contrast, a red-shifted profile is one in which the peak intensity is in the red wing. A red-shifted profile can be unambiguously interpreted as a Doppler shift representing motion away from the observer and hence is the more relevant parameter for this discussion. Teske (1962) was the first to find a clear example of a red-shifted profile in $\mathrm{H} \alpha$ spectra taken at the McMath Hulbert Observatory. A typical red-shifted profile at the growing boundary of a two-ribbon flare is illustrated in Švestka, Martin, and Kopp (1980). An unusually clear example of a flare profile with a large red-shift is seen in Figure 20 of Zirin and Tanaka (1973). More typical examples are shown in Figure 1 of Ichimoto and Kurokawa (1984).

In their comprehensive study of flare spectra, Ichimoto and Kurokawa (1984), unlike the previous authors, used the terms red-shift and red asymmetry interchangeably but they primarily show examples of red-shift. This usage of terms seems justifiable if the red asymmetry comes from a combination of red-shifted and non-shifted profiles which are not spatially resolved. In specific studies, such as those of Teske (1962) and Švestka, Martin, and Kopp (1980), it is clear that flare elements of medium and faint intensity do not exhibit red-asymmetry but do exhibit red-shift. At this writing, it is still not evident, from previous studies of bright flares and flare kernals, whether the redasymmetry results from a combination of unshifted and shifted profiles or from the Stark-effect or other phenomenon independent of red-shift (Švestka, 1976). However, Ichimoto and Kurokawa (1984) interpret all red-asymmetry and red-shift as downward motion.

Studies from filtergrams alone do not allow one to distinguish between red-asymmetry and red-shift, but they do give a more complete picture of the evolution of flare elements than most flare spectra with the exception of time-lapse spectra. Studies of time-lapse spectra (Ichimoto and Kurokawa, 1984), and studies from time-lapse filtergrams of mostly chromospheric flare elements (Tang, 1983), show the red-shifts (or red-asymmetries) of individual flare elements to be a time and energy dependent characteristic of the flare fine structure rather than of the flare as a whole. When fine structure can be resolved in flares, it is seen that chromospheric flares consist of a succession of many elementary localized brightenings. Even what we call 'flare kernals' are usually a whole envelope of these successively forming and decaying flare elements. This is exemplified in the flares of 5 November, 1980 illustrated in Wu et al. (1986). Only minutes after a flare element forms, its red-shift disappears and is supplanted by a nearly symmetric flare profile. The red-shift occurs only while the flare or any specific part of a flare is increasing in optical intensity (Tang, 1983; Ichimoto and Kurokawa, 1984). 


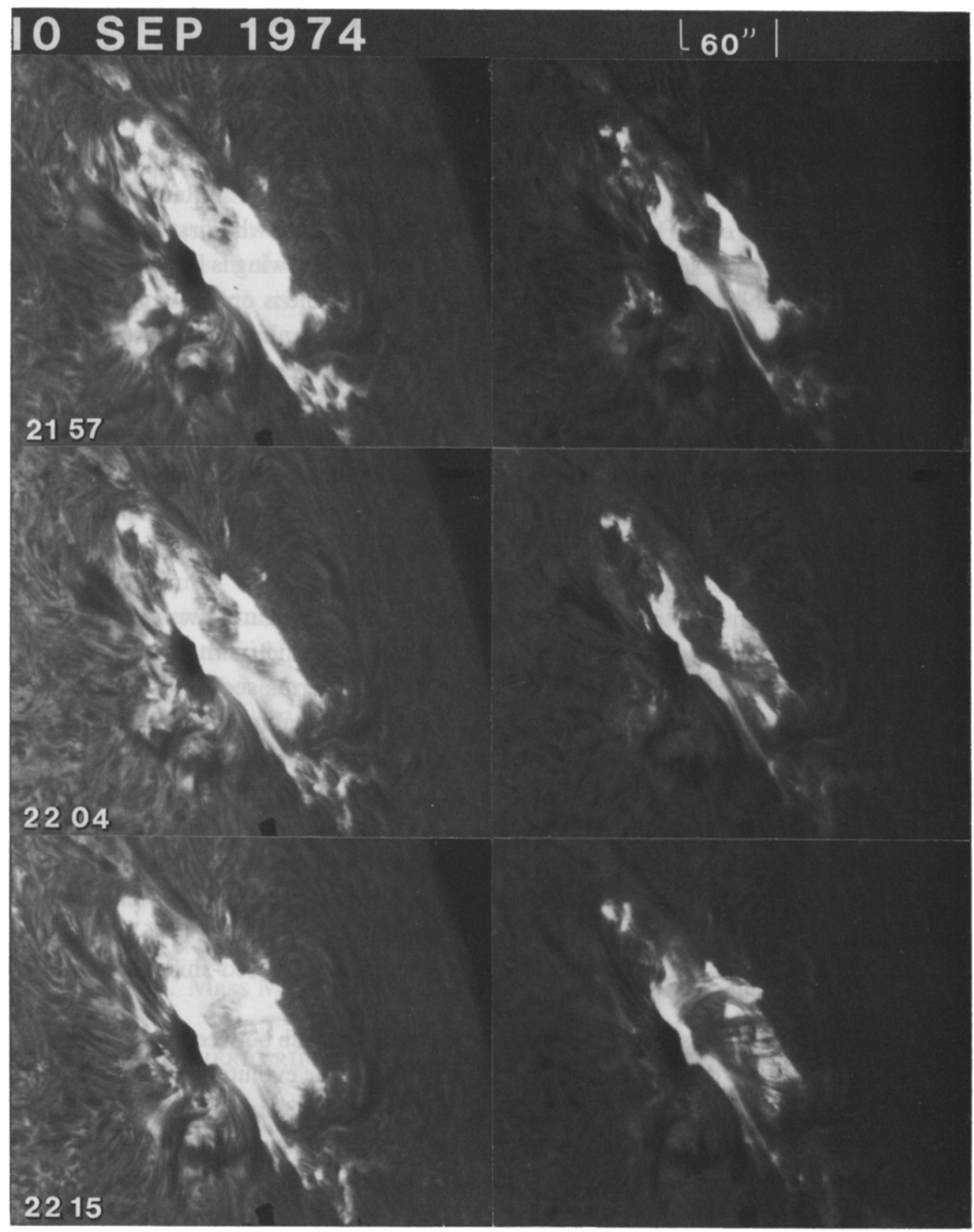

Fig. 1a. The early development of flare loops are shown in normally exposed prints on the left and in heavily exposed $(\times 4)$ prints at the same times on the right. In the heavily exposed prints, the near legs of the loops appear as approximately parallel, dark striations superposed against the chromospheric flare ribbons.

The red-shift is most clearly seen in spectra of flares that cover large areas of the chromosphere (Švestka, Martin, and Kopp, 1980). In such flares, it is also clear that the red-shifted flare elements occur only in newly formed flare elements at the outer perimeter of the flare and occur simultaneously on both sides of the polarity inversion 


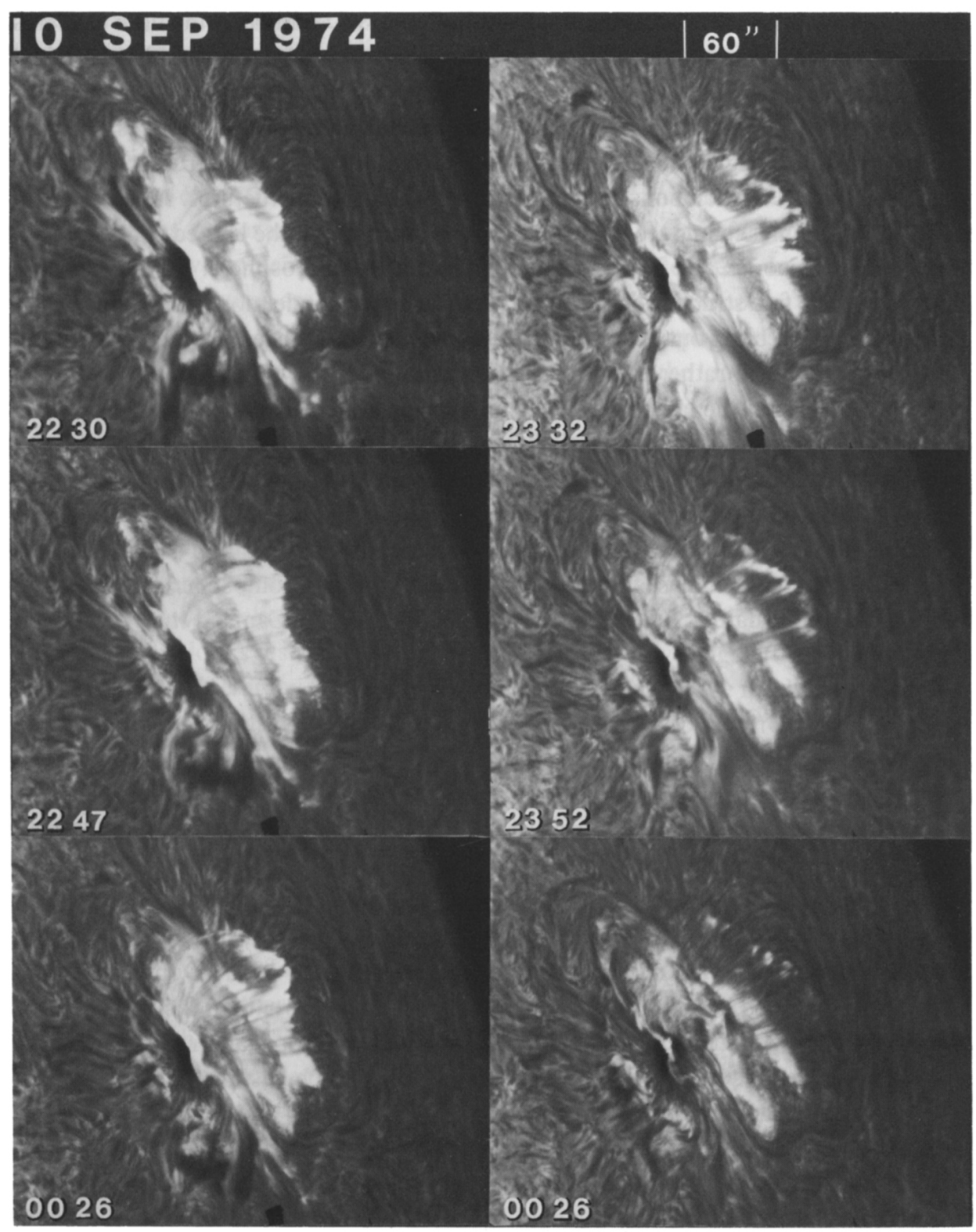

Fig. 1b. As the chromospheric flare ribbons separate, the rising system of loops, that connect the ribbons of opposite polarity, become more conspicuous at normal exposures for the chromosphere. The tops of the loops have concentrations of emission while the legs are less bright and appear in absorption against the flare ribbons.

line that separates the flare ribbons (Rust and Bar, 1973). Because a whole flare does not form simultaneously, it is difficult (and often inaccurate) to describe flares as having 'phases'. Even during the so-called 'decay phase' of extensive flares, newly forming 
red-shifted flare elements continue to occur (Tang, 1983). Similarly, the flare elements that form early in a flare can already be decaying before the flare as a whole has reached maximum intensity or area.

The accuracy of description, and hence the physical interpretation of flares and their mass motions, are highly dependent upon the resolution of the fine structure of flares. It is likely that all flare ribbons are composed of sub-arc sec structures. However, very often photographic techniques limit its visibility in addition to the limited spatial resolution of our telescopes and image degradation from our atmosphere. As illustrated in Figure 1(a), the fine structure in flares can sometimes be made more visible by heavily exposing prints to bring out detail captured on film which has been exposed to optimize chromospheric detail rather than detail in flares.

As will be seen in the later sections of this paper, the mass motions of the chromospheric flare ribbons differ substantially from the mass motions of the coronal components. Chromospheric flare elements have a very small range of redshifts while the associated coronal components such as erupting filaments and surges typically reveal both blue shifts and red shifts and have a much higher velocity range. These differences are exemplified in the analyses of the energy content in the chromospheric and coronal components of the flare of 5 September, 1973, respectively, by Canfield et al. (1980) and Webb et al. (1980).

\subsection{REMOTE CHROMOSPHERIC FLARE COMPONENTS}

Some flares also have peripheral chromospheric flare components in addition to the ribbons that are centered around a polarity inversion zone. These peripheral flare patches have previously been designated as 'remote brightenings' (Tang and Moore, 1982). They can be tens to a few hundred thousand kilometers from the primary flare site. To my knowledge there are yet no published papers that give Doppler shift measurements of these peripheral parts of flares. It is expected, however, that these remote brightenings would not have Doppler shifts in excess of the Doppler shifts of the flare kernals. In many circumstances it has been shown that the remote flare components derive their energy from the source flare via the propagation of a disturbance along coronal arches connecting to the source flare. Such disturbances have been detected as: (1) flaring arches-mass flowing in coronal arches and seen in emission in $\mathrm{H} \alpha$ in projection against the disk (Mouradian, Martres, and Soru-Escaut, 1983) following the flow of X-ray emission (Martin and Švestka, 1988) or (2) soft X-ray emission with no visible emission in $\mathrm{H} \alpha$ (Rust and Webb, 1977; Tang and Moore, 1982), or (3) radio emission (Tang and Moore, 1982).

\section{Coronal Flare Components}

\subsection{FLARE LOOPS}

Flare loops in $\mathrm{H} \alpha$ are interpreted as just the late phase of flare loops that form at the ouset of a flare (Moore et al., 1980). The X-ray experiments on board Skylab firmly 
established that flares in X-rays, almost invariably have the form of coronal loops that link the positive and negative chromospheric flare kernals and ribbons (summary by Sturrock, 1980). The spatial resolution of most X-ray images of flares, acquired to date, does not allow us to resolve the many tightly-packed, individual loops that must exist as a flare rises to maximum intensity. However, we know that this finely-structured system of loops exists from observations in $\mathrm{H} \alpha$ during the maximum and post-maximum phases of flares. An example of flare loops is shown in Figure 1(a); to better identify the loops, the development of the flare is shown at 2 print densities. In the left column of images, the exposures are made to show the chromospheric structure and the general outline of the flare. In the right column of images, longer exposures, by a factor of 4 , reveal the early appearance and fine structure of the legs of the loop system superposed against the rest of the flare. As the brightness of the flare declines, as shown in Figure 1(b), the loops become apparent at print densities appropriate for the chromosphere. Because the site of this flare is quite near the limb, it can be seen that new loops form at successively higher altitudes. Less apparent, in these still images, than in the time-lapse movie of this event, is the continuous downflow of mass from the tops of the loops to the flare ribbons below. At this stage, the loops actually connect the inner decaying part of the chromospheric flare ribbons (Rust and Bar, 1973). Above these loops in $\mathrm{H} \alpha$ are loops visible in X-rays, EUV, and UV wavelengths. The X-ray loops are the top of the hierarchy (Moore et al., 1980). They connect the newly-forming outer parts of the flare ribbons while the EUV and UV wavelengths connect to the intermediate parts of the ribbons.

It is common for the chromospheric flare ribbons to appear to move away from each other with time. This is an apparent rather than a real motion that is due the new flare elements forming on the outer boundaries of the flare ribbons concurrently with the formation of the coronal flare loops in X-rays (Rust and Bar, 1973; Moore et al., 1980).

In $\mathrm{H} \alpha$ the loops typically reveal downflows in both legs of the loops in the range of $50-100 \mathrm{~km} \mathrm{~s}^{-1}$. Downflows of this magnitude have little or no ability to depress or brighten the chromosphere. Flare spectra confirm the absence of red-shift during the late phase (the $\mathrm{H} \alpha$ phase). The red-shifts, discussed in Section 2.1, occur concurrently with the formation of the loops in X-rays. This implies that either high speed particles and/or possible shock waves are responsible for the red shifts that occur during the early development of the chromospheric flare elements. The loops in $\mathrm{H} \alpha$ are only an aftermath of a much more energetic process. From the presence of the flare loops in $H \alpha$, we can also deduce that another invisible phase of mass motion must take place between the initial phase which causes the red-shifted chromospheric elements and the late phase when $\mathrm{H} \alpha$ loops are seen. The $\mathrm{H} \alpha$ loops are too dense for their mass to be condensed out of the corona. Therefore, the filling of the loops with dense mass must be initiated at their footpoints; such upflows have recently been observed in $\mathrm{H} \alpha$ Schmieder et al. (1987). Substantial evidence of the upflow of mass into flare loops has also been observed at shorter wavelengths as discussed in Doschek et al. (1986). Thus flare loops have at least three stages of mass motions: (1) an inferred rapid downflow of high speed, possibly relativistic particles, (2) an upflow, now often called 'chromospheric evapo- 
ration' in the range of $100-500 \mathrm{~km} \mathrm{~s}^{-1}$, and (3) a slow visible downflow commonly observed in $\mathrm{H} \alpha$ in the range of $50-100 \mathrm{~km} \mathrm{~s}^{-1}$ (and other chromospheric lines) as illustrated in Figures 1(a) and 1(b).

For flares that occur very close to the limb, the loops are readily observed against the background of the sky through appropriate filters.

However, loops in $\mathrm{H} \alpha$ are only seen against the disk if the densities of the loops reaches approximately $10^{11} \mathrm{~cm}^{-3}$. Because of this density requirement, only a minority of flares actually exhibit flare loops in $\mathrm{H} \alpha$ in projection against the disk. Usually these are large and bright flares in $H \alpha$. In the less dense loops or in the legs of the loops, the loops appear in absorption rather than emission. In a few flares, the tops of the loops remain bright throughout their whole evolution. However, it is more common to see the

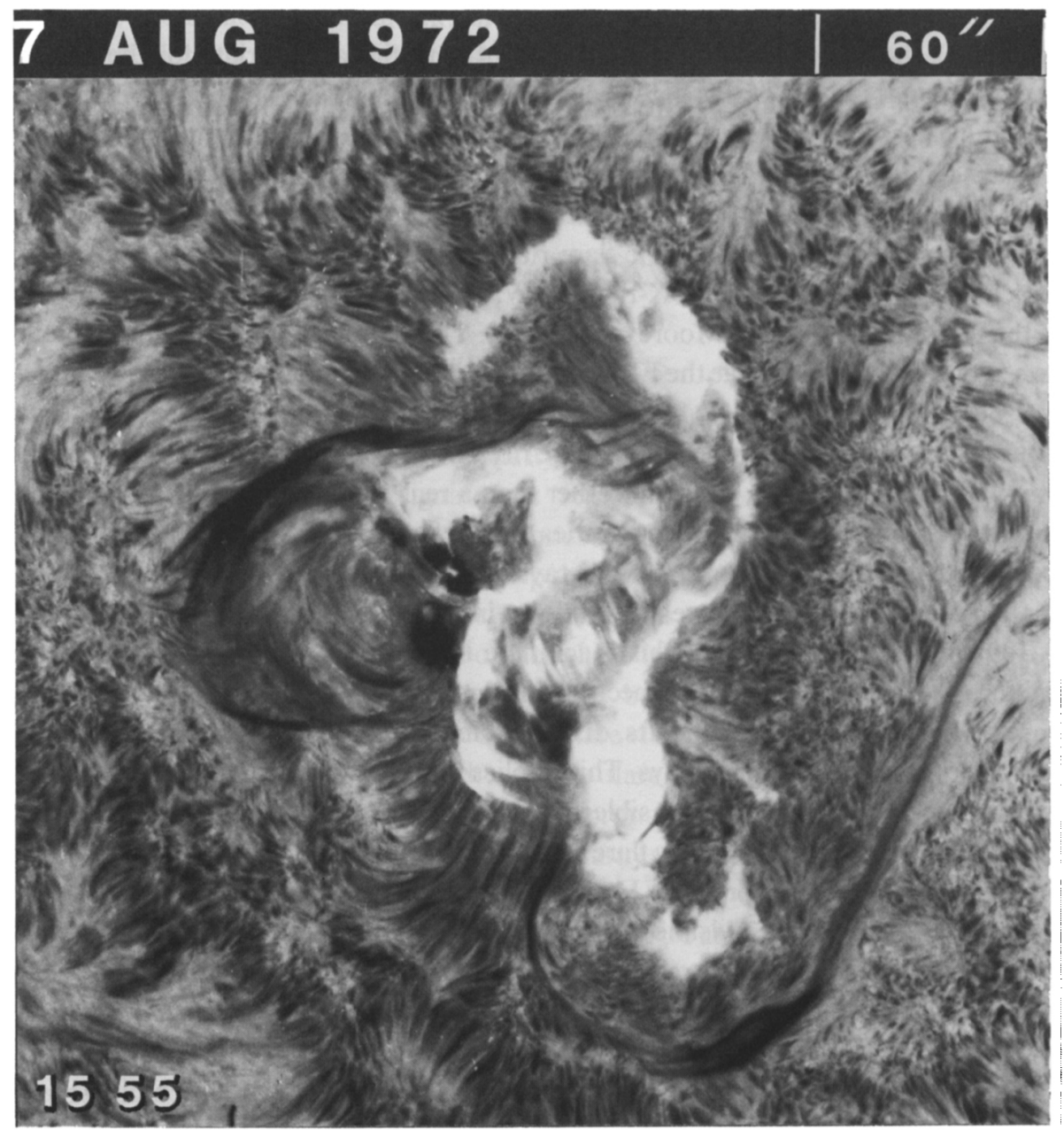

Fig. 2. Flare loops both in absorption and in emission extend between the chromospheric flare ribbons. In this case we see only the tops of the loops. The legs and footpoints of the loops cannot be seen because their Doppler shifts exclude them from the passband of the filter used to take these images. The filter passband was $0.25 \AA$ centered at approximately $\mathrm{H} \alpha-0.5 \AA$. 
loops gradually change in time from emission to absorption or to see them only in absorption (Martin, 1979) in projection against the disk.

A seen through narrow band $\mathrm{H} \alpha$ filters $(0.5 \AA$ or less $)$ in line center, one rarely sees the entire loop because the Doppler shifts are usually in excess of the bandwidth. However, with a rapidly tunable filter, it is possible to observe the full range of loop velocities. If viewed near disk center, only the tops of loops are typically observed such as in Figure 2. In this example, some of the loops are in emission and another set are in absorption and many others are invisible. Because the density of the successively formed loops decreases rapidly with time, they become invisible, in projection against the disk, usually before the chromospheric ribbons have decayed. In observations at the limb, however, it is well known that the loops often are observed many hours after the chromospheric elements have disappeared in $\mathrm{H} \alpha$.

\subsection{Flaring ARCHES}

The term 'flaring arches' was introduced by Mouradian, Martres, and Soru-Escaut (1983) to refer to parts of flares that have not commonly been discussed in the literature. Martin and Švestka (1988) and Mouradian and Soru-Escaut (1989, this Colloquium) have more specifically defined the term flaring arches to describe a component of some flares in which emitting mass is observed first to flow upward into the corona, traverse an arch-like path, and descend to another point in the chromosphere. An example, seen in projection against the disk, is illustrated in Figures 3(a) and 3(b). Figure 4 shows a flaring arch at the limb. The energy for the upflow is somehow derived from processes associated with the formation of flare loops and ribbons.

Flaring arches can vary over a wide range of dimensions and intensities at the observed wavelengths. Martin and Švestka (1988) reported that flaring arches have an $\mathrm{X}$-ray phase, usually preceding the bulk of $\mathrm{H} \alpha$ mass that travels through the arch. They also found the early excitation of the secondary footpoint seen both before the arrival of the $\mathrm{H} \alpha$ mass, and surprisingly, even before the arrival of the first detectable hard $\mathrm{X}$-rays. But it should be noted that additional brightenings of the secondary footpoint were also seen at the time the X-ray emission reached the secondary footpoint.

The complex example in Figure 3(a) shows a series of arches and a series of secondary footpoints rather than a single arch and a single secondary footpoint as in the events described by Martin and Śvestka (1988) and Švestka et al. (1989). The flare is in progress in the first frame of Figure 3(a) and an ejection of mass can be seen protruding from the flare. In successive frames, it is seen that the mass ejecta follows a curved trajectory and intersects the chromosphere first at P1 at a distance of about 1 arc min from the primary footpoint, and subsequently, at P2 and P3, about 2 arc min from the source. Note that P1, P2, and P3 all brighten before the successive appearances of visible mass in the various arches. Following the initial bright ejecta confined to the arch system connecting to $\mathrm{Pl}$, less bright mass continues to pour from the same initial site towards P2 and P3. This mass is seen partly in emission and partly in absorption in frames at 19:37 and 19:45. Because the closest part of the limb is toward the top of the frames, the longer absorption arches apparently also have a higher trajectory than 
the initial arch in emission. Although there were no spatially resolved X-ray images acquired, we suspect from the similarity of this event to the ones already studied, that the visible ejection is undoubtedly preceded by $\mathrm{X}$-rays that traverse the arches at a higher

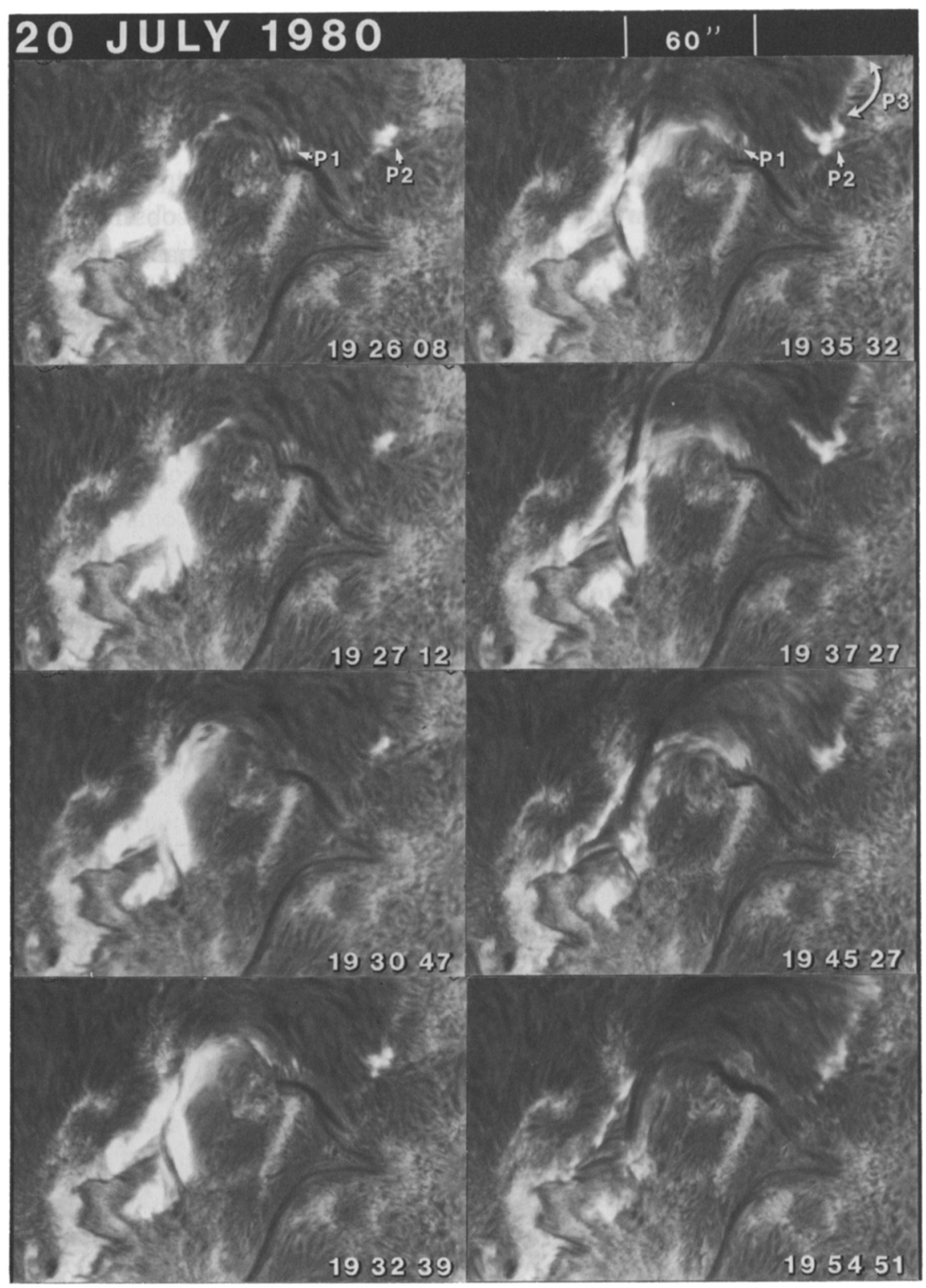

Fig. 3a. The beginning of the flaring arch is the emission protruding upward from the flare in the first frame. In successive frames in the first column, this emission follows an arch-shaped trajectory through the corona to a chromospheric footpoint, $\mathbf{P 1}$, approximately 1 arc min to the upper right the primary flare site. The emission phase of this 'flaring arch' is followed by the flow of additional mass, mostly seen in absorption, from the flare site to more distant chromospheric footpoints, P2, and adjoining row of fainter footpoints, $P 3$, in the upper right of each frame. 
speed than these $\mathrm{H} \alpha$ components; the X-ray arches are expected about the same time as the brightening of the secondary footpoints.

Figure 3(b) illustrates the Doppler shifts in the same flaring arches on 20 July in the wavelength range from -1 to $+1 \AA$. In the first row are images at the center of the $\mathrm{H} \alpha$ line and in the second and third rows are images showing Doppler-shifted components at - and $+0.5 \AA$ and - and $+1.0 \AA$, respectively. Blue wing images showing the upflow are on the left and red wing images showing the downflow towards the secondary footpoint are on the right.

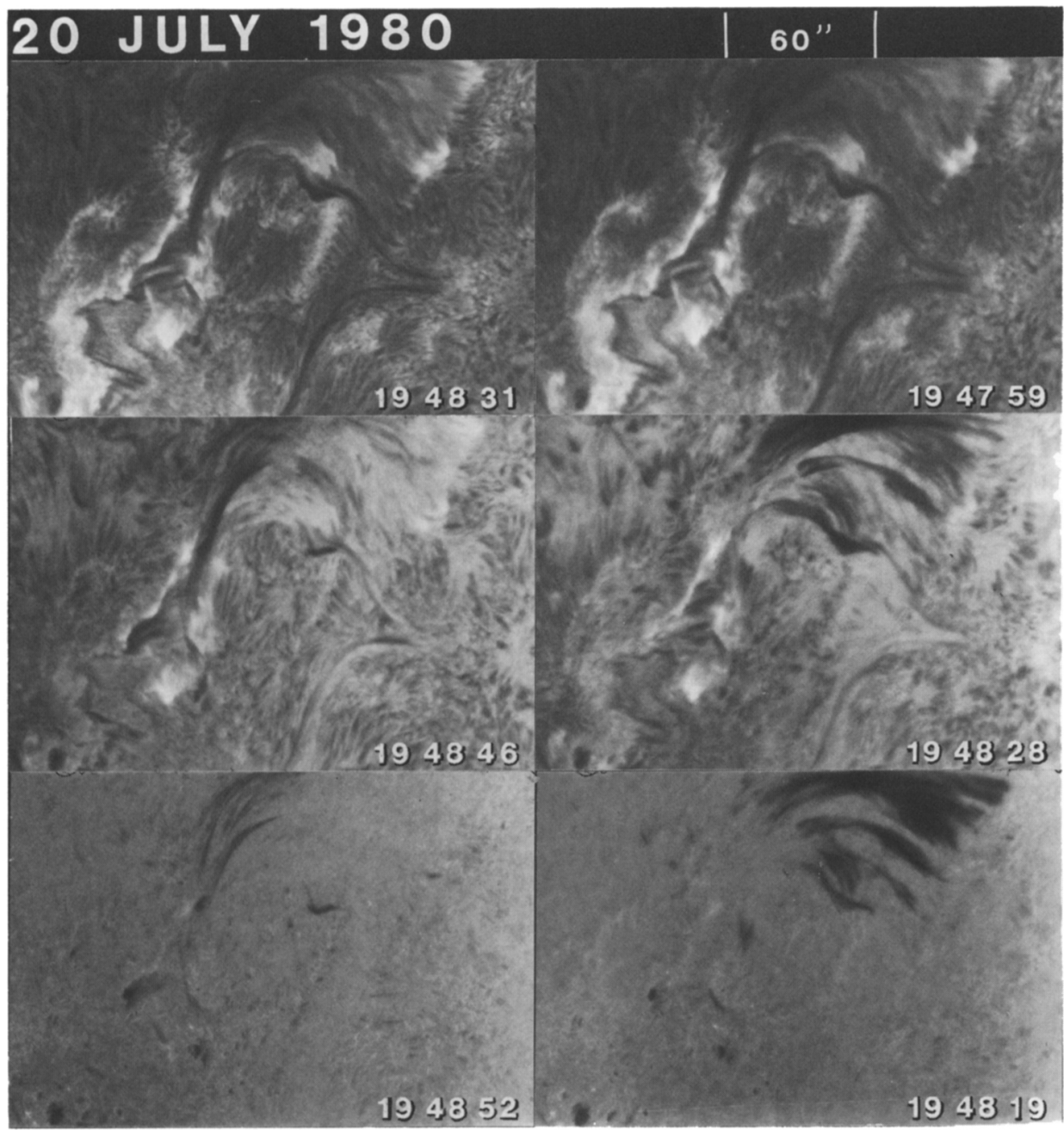

Fig. 3b. The Doppler-shifted mass within the same flaring arches as in Figure 3(a) is illustrated in the first column in images at $\mathrm{H} \alpha$ centerline, $\mathrm{H} \alpha-0.5 \AA$ and $\mathrm{H} \alpha-1.0 \AA$ and in the second column at $\mathrm{H} \alpha$ centerline, $\mathrm{H} \alpha+0.5 \AA$ and at $\mathbf{H} \alpha+1.0 \AA$. This series of images, taken in a two-minute interval, shows the pattern of Doppler shifts at about the same time during the event. They confirm the visual impression seen in the time-lapse film of upward motion from the main flare site along an arch-shaped path and then downward to the distant secondary footpoints in the upper right of each frame. 
An example of a flaring arch at the limb is shown in Figure 4 in images recorded at the former observing site of the Lockheed Solar Group. The limb observations were made through a $10 \AA$ interference filter. The event has a duration of about one hour although the full extent of the arch is seen about 20 minutes after the start of the event. The source flare is not seen but that is not surprising because it is probably small and

\section{JULY 1966}

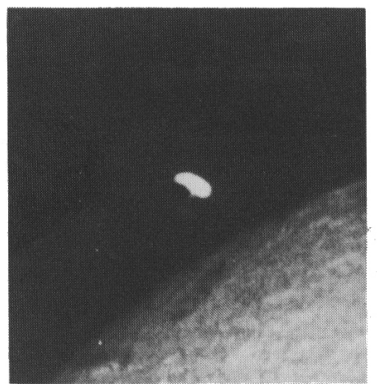

2321:00

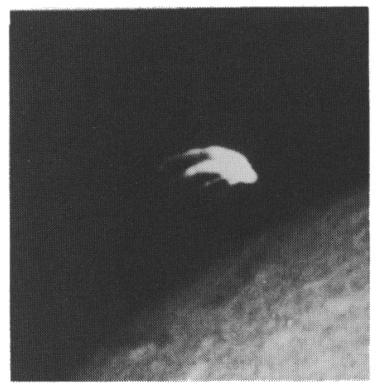

$2336: 30$

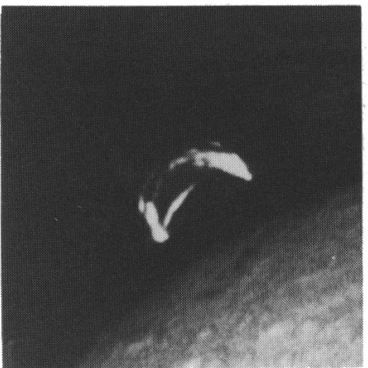

$2349: 00$

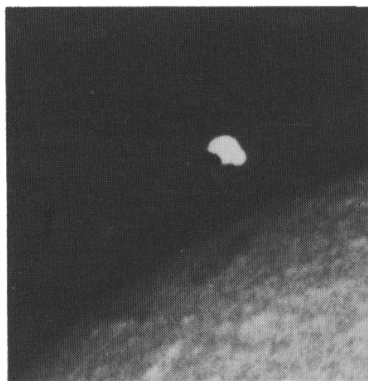

$2330: 00$

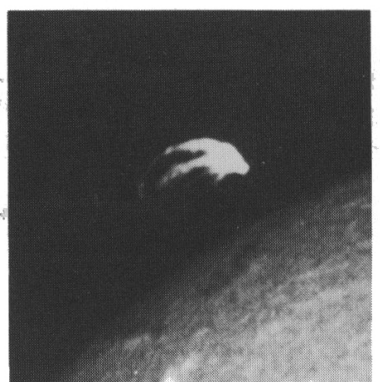

$2338: 30$

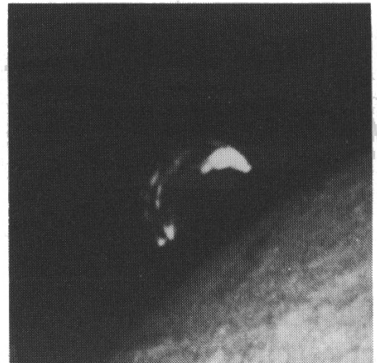

$2353: 00$

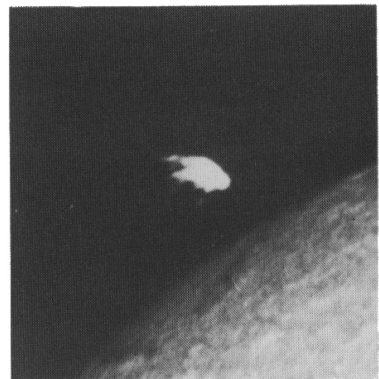

$2324: 30$

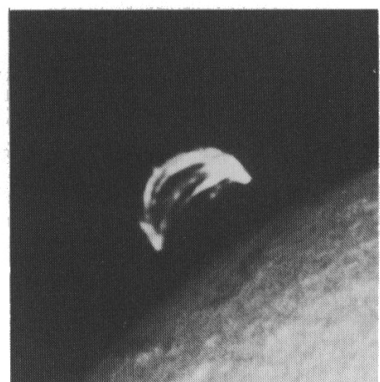

$2342: 30$

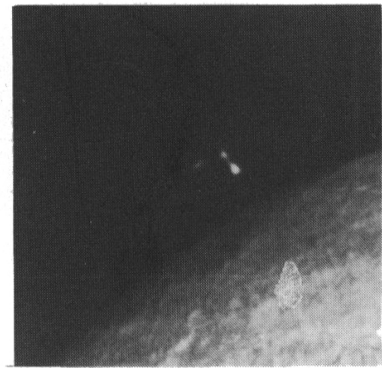

$0021: 00$

Fig. 4. This example of a flaring arch at the limb was photographed through a $10 \AA$ filter at the former site of the Lockheed Solar Group at Saugus, California, U.S.A. The disk of the Sun, however was photographed through a filter of $0.5 \AA$ passband. The mass in the flaring arch travels from right to left. 
obscured by the occulting disk. This event is remarkably similar to the 6 November, 1980 event published by Martin and Švestka (1988) which was associated with a very small source flare.

Flaring arches and flare loops have been shown here to be very different in their pattern of mass motions and in their spatial relationship to the polarity inversion line separating the flare ribbons. Surges on the other hand are more similar to flaring arches than they are different. Both flaring arches and surges follow pre-existing lines of force - unlike flare loops systems (Martin, 1979) - and both can occur in homologous series on the order of tens of successive events.

\subsection{SURGES}

Surges are the most common of all flare-associated mass motions. Surges are traditionally defined as spike-shaped mass ejecta that move from the chromosphere into the corona, slow with time, stop, and flow back to the chromosphere in the reverse direction from their outflow. This definition is somewhat too restrictive to fit all surges and should be understood as a very general definition rather than an exact definition. Exceptions are observed in which not all of the surge mass falls back to the originating site. Additionally, the paths of outflow and subsequent inflow are generally parallel but not necessarily coincident. Occasionally, some of the mass of a surge, like a flaring arch, is seen to traverse the entire distance of a coronal arch from the initiating site to a secondary site. Like flaring arches, surges can be entirely in emission, on part in emission and part in absorption, although most surges are seen entirely in absorption. Like flaring arches, surges also tend to follow existing lines of force and they most frequently occur in homologous series.

Figure 5 displays an example of a surge that is in emission when it begins (first 3 frames, 22:30-22:35, arrow points to surge). Thereafter it gradually becomes an absorbing feature as seen projected against the chromosphere. The outflow phase lasts until $\sim 22: 45$. The inflow phase becomes conspicuous at 23:02. This surge is terminated by the next homologous surge originating from nearly the same site at $\sim 23: 12$.

Although some studies have found an absence of X-rays specifically at the sites of surges (Schadee and Martin, 1986), other studies have found emission in the UV and X-rays to be coincident with surges (Rust, 1980; Rust et al., 1977; Schmieder et al., 1988). From the differences in these studies, it seems that surges in absorption are probably at the opposite end of the energy spectrum from surges in emission. Surges in absorption are unlikely to have related X-ray emission while surges in emission are likely to have $\mathrm{X}$-ray emission. There has been no study of just bright surges to find if they are generally associated with X-ray or UV radiation. The study of Rust et al. (1980) indicates that many surges might have a UV component.

As noted above, there are minimal differences between flaring arches and surges in emission; the major difference for flaring arches is that the dense ejected mass successfully reaches the top of a pre-existing coronal loop and hence some or all of the mass falls (or is driven) down the other end of the loop instead of returning to its initial site. I, therefore, suggest that flaring arches are the equivalent of 'super' surges. A principal 
question that needs to be addressed about surges (and flaring arches) is how their energy is derived.

Although it is sometimes stated that surges are not always associated with flares such as in the paper by Schmieder et al. (1988), the question of flare association has much

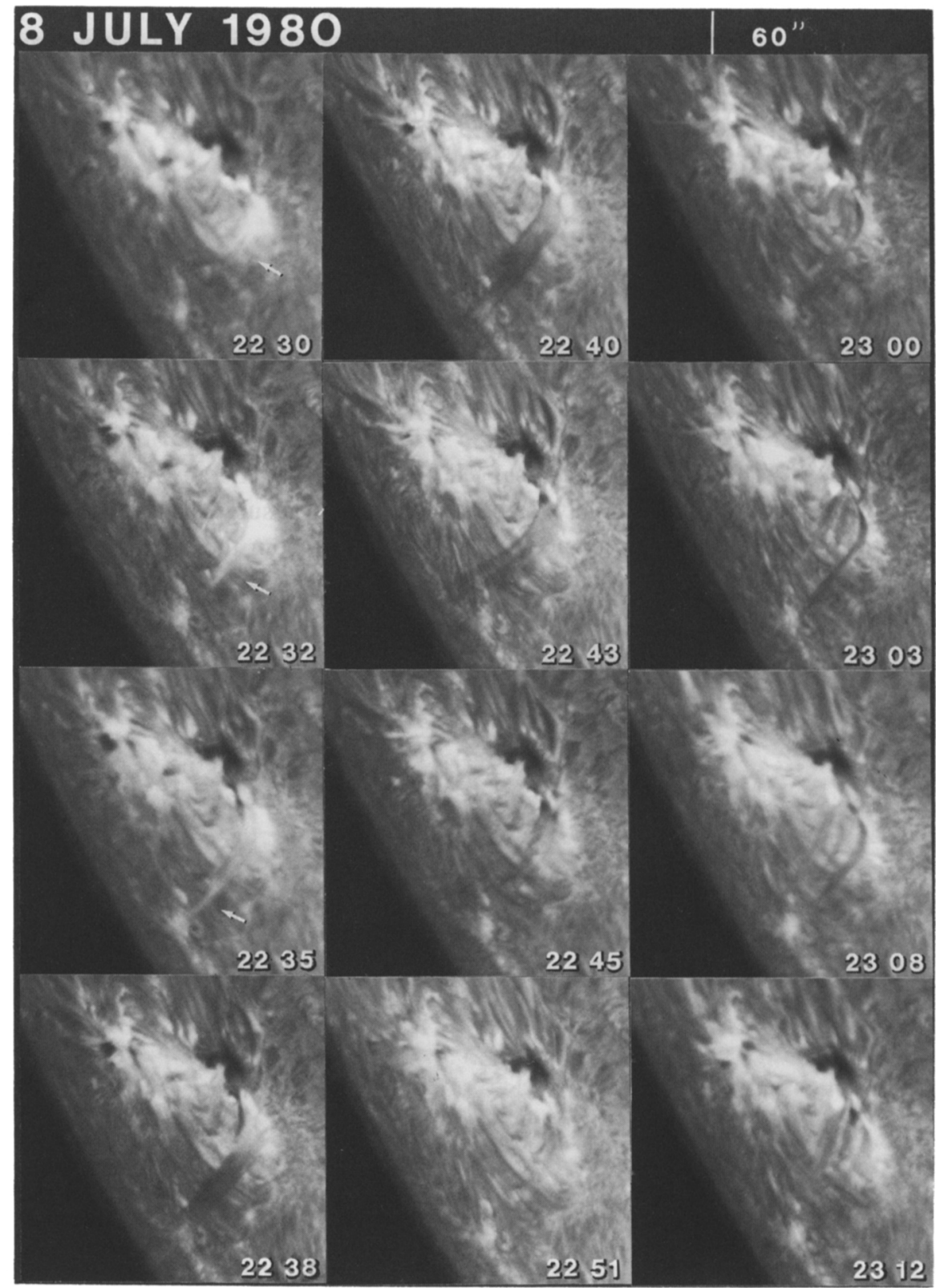

Fig. 5. The arrows in the first 3 frames point to a surge in emission. As the mass extends into the corona, the surge gradually changes from emission to absorption. Surges seen in emission occasionally have been recognized in UV and X-ray images. 
to do with how one defines both a flare and a surge. In some cases, the flares at the base of a surge may be very small in comparison with the associated surge or even obscured by the surge or mistaken as part of the surge.

\subsection{ERUPTING FILAMENTS AND FLARE-ACTIVATED FILAMENTS}

Three classes of activated filaments initiated by or with flares are discussed and illustrated:

(1) the preflare activation and subsequent eruption,

(2) the surge-like activation,

(3) activation via impact from ejected mass or a shock wave produced by a neighboring flare.

The mass motions associated with each of the above types of activations follow different patterns.

The first category is the only one that should be considered a 'primary' activation because it precedes and accompanies the emission components of a flare in a pattern that suggests that it is an intimate part of the process of energy release of the flare. Hence, this category of activation is sometimes considered (as in this paper) to be an integral part of the flare process while the other categories are secondary processes that occur after or because of the primary energy release.

The 'preflare activation and subsequent eruption' is a continuous process that is often seen to begin during the hour preceding the initiation of the flare loops and chromospheric ribbons. The preflare activation is an outward expansion of either the middle or one end of a filament usually accompanied by increased absorption. The preflare activation irreversibly leads to the complete eruption of the filament, and usually to the expulsion of some of its mass into the interplanetary medium.

The preflare activation and eruption of filaments with major flares is described in detail by Smith and Ramsey (1964) and statistics are presented in Martin and Ramsey (1972). Specific examples are cited in a review by Martin (1980). Relationships of the preflare activation to other preflare conditions and changes at other wavelengths are discussed in Schmahl et al. (1986) and by Gaizauskas (1989, this issue).

Three widely differing examples of the preflare activation and eruption of filaments are shown in Figures 6 and 7. The first 4 frames of Figure 6 (1st column) show the slow preflare ascent of a filament observed on 7-8 June, 1985. The preflare component of motion in the plane of the sky can be seen in Figure 6 only in the upper (southern) end of the filament from 00:05 until the start of the flare at 00:15. As the outward motion of the filament accelerates during the flare, it is clear that the southern end of the filament is rising more rapidly than the northern end. This leads to a 'whip-like' eruption as opposed to the also common 'symmetric' eruption in which the middle of a filament rises while both ends temporarily retain a connection to the chromosphere. The chromospheric connection is maintained as long as there is mass flow down one or both legs of the filament. During the whip-like eruption in Figure 6, the more rapidly ascending end of the filament disconnects with the chromosphere while downflow of some of the mass of the filament simultaneously occurs in the other end of the filament. The 
combination of bodily outward motion and internal downflow keeps this lower leg of the erupting filament (EF) visible until 00:32 in Figure 9. The disappearance occurs as successive parts of filament exceed the Doppler shifts that are visible within the filter

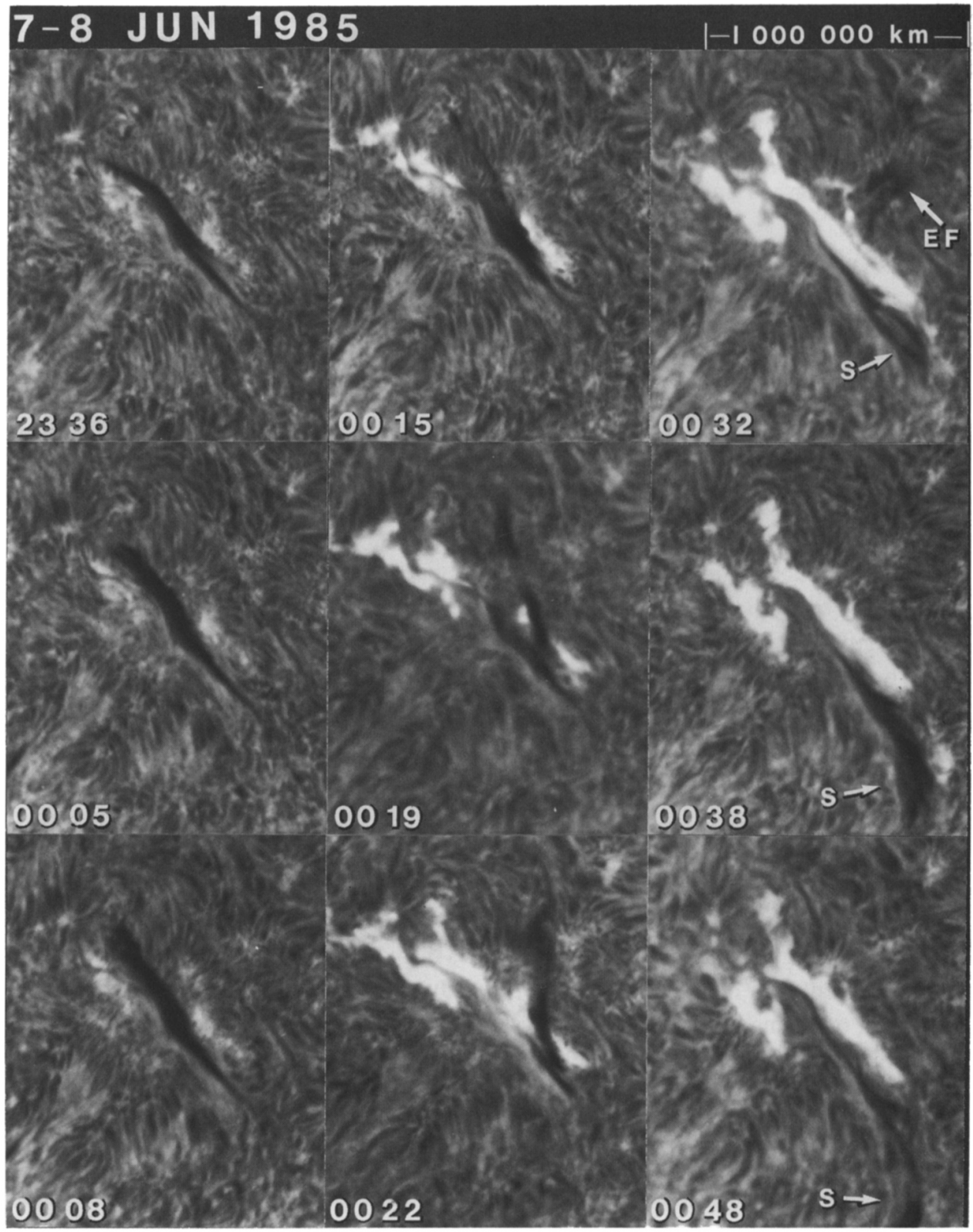

Fig. 6. This example of an erupting filament with a flare has the form of a whip as the upper end ascends more rapidly into the corona than the lower end. A non-erupting low segment of the filament is ejected as a surge. This mass subsequently flows back into the polarity inversion zone (the channel or site previously occupied by the erupted filament), where it contributes to the formation of a new filament. 


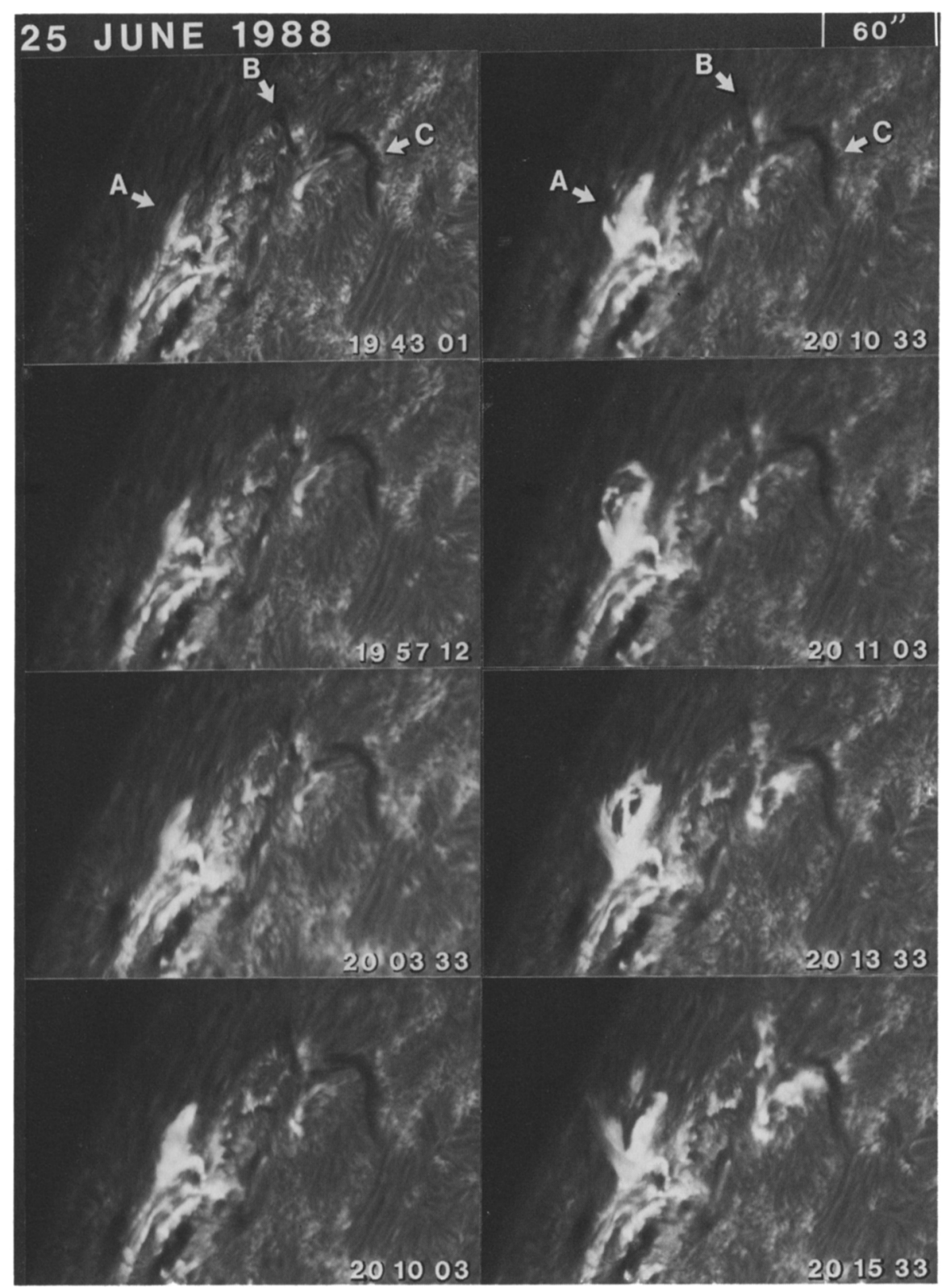

Fig. 7a. The two major flares in this series are each accompanied by the eruption of the filaments labelled $A$ and $B$ in the top row. Although filament $A$ appears to be of flare intensity, the corresponding images at $\mathrm{H} \alpha-0.6 \AA$ in Figure 7(c) show the filament to be a separate structure from the smaller underlying chromospheric flare. Filament $B$ also changes from an absorbing feature to an emission feature in $\mathrm{H} \alpha$ as it erupts. A mass ejecta from the flare associated with erupting filament $B$ impacts filament $C$ at about $20: 15$, the last frame.

passband. In the case in Figure 6, the passband of the filter is $0.25 \AA$ (halfwidth at half maximum) centered at approximately $0.25 \AA$ in the blue wing of the $\mathrm{H} x$ line. The mass 


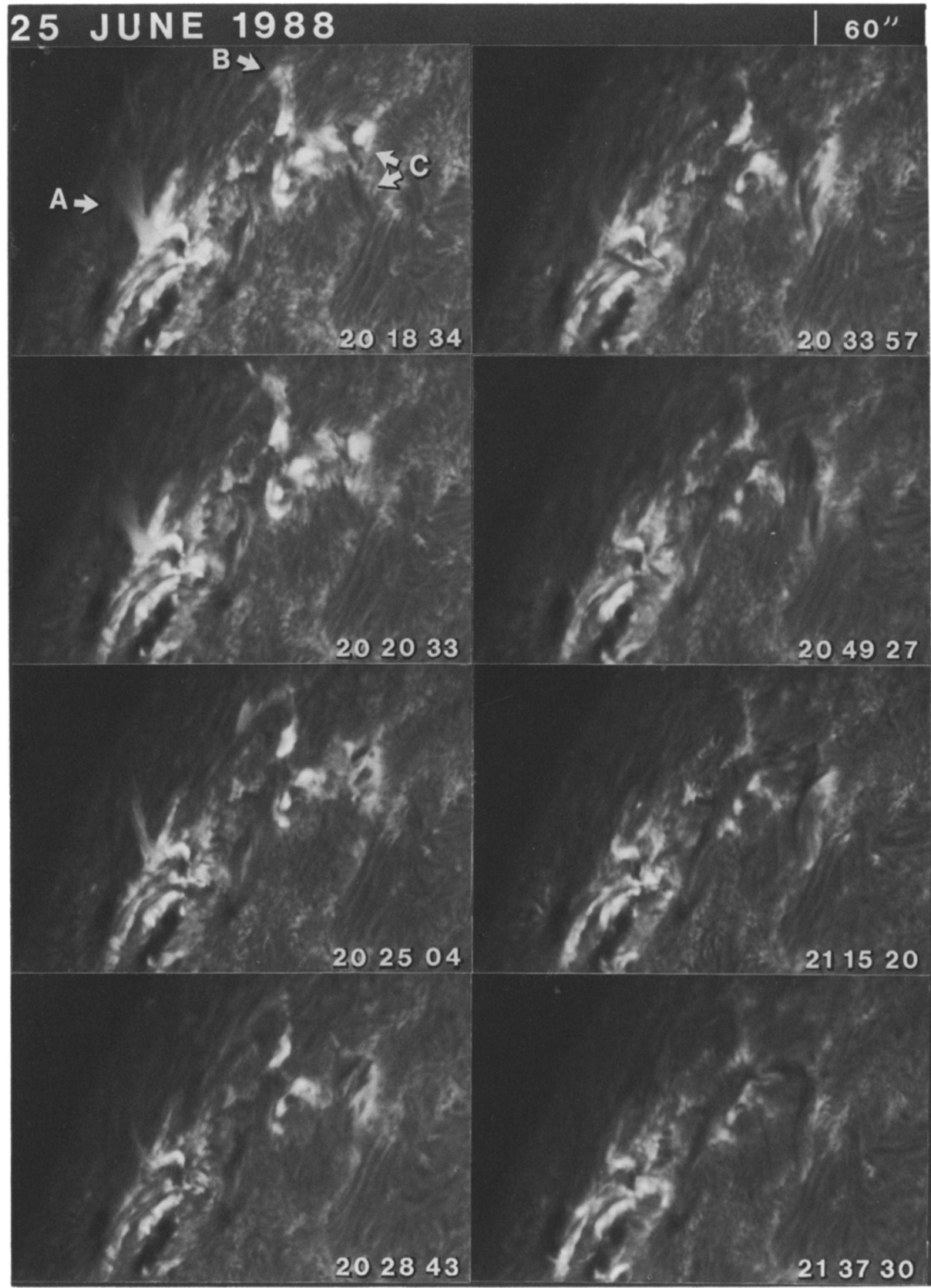

Fig. 7b. The erratic activation of filament $C$ is seen to begin abruptly after the impact of a mass ejection from the flare associated with erupting filament $B$. After about one hour of turbulent motion, filament $C$ assumes its approximate former configuration.

of the filament is visible in the approximate wavelength range of twice the half-width of the filter, or from 0 to $-0.5 \AA$, corresponding to a maximum line of sight velocity of $45 \mathrm{~km} \mathrm{~s}^{-1}$. 


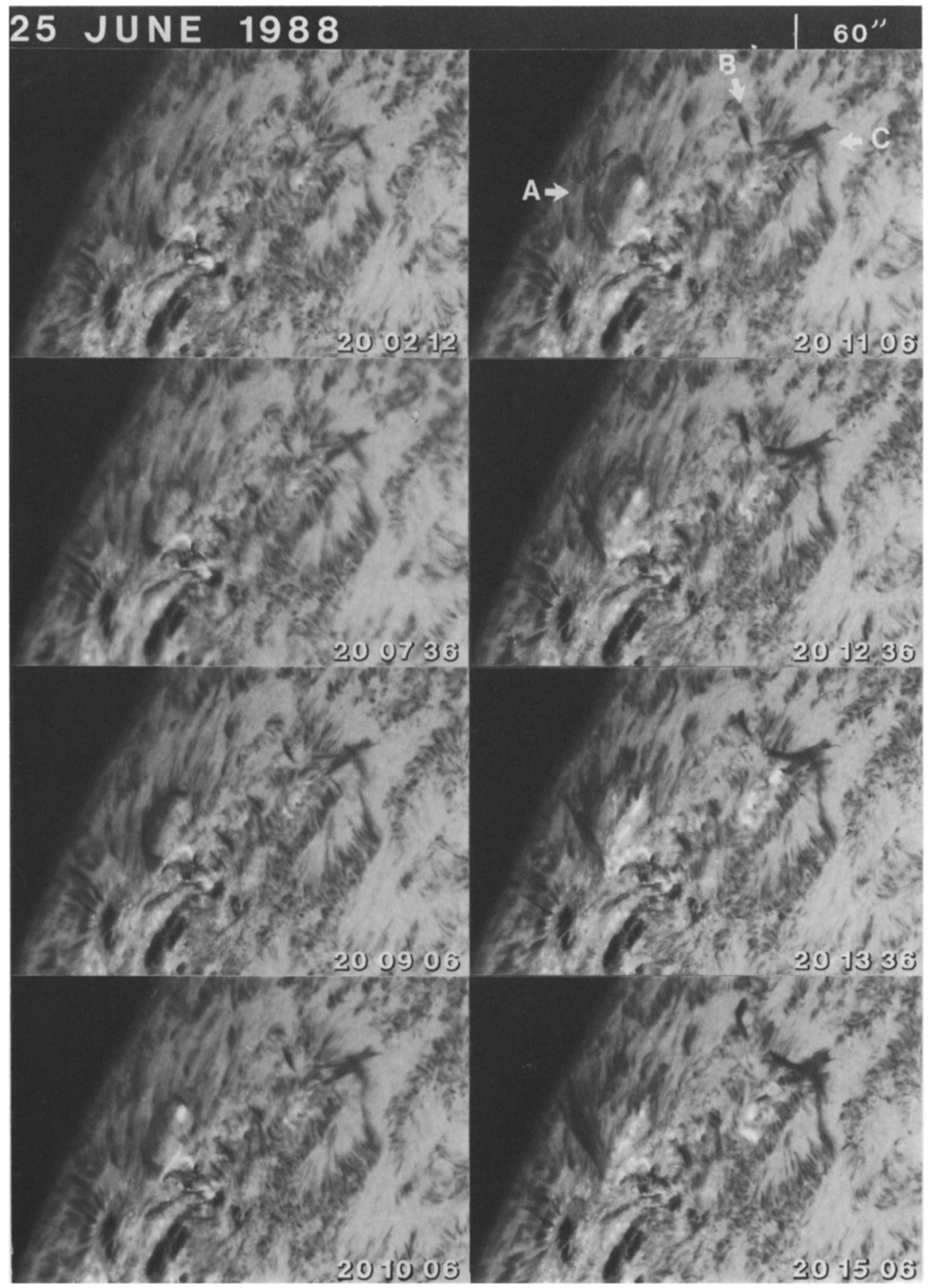

Fig. 7c. These images at $\mathrm{H} \alpha-0.6 \AA$ allow one to more clearly distinguish the activated filaments $A$ and $B$ from other chromospheric features than in the $\mathrm{H} \alpha$ centerline images in Figures $7(\mathrm{a})$ and $7(\mathrm{~b})$; the active filaments have high contrast relative to the chromosphere and most of the flare emission cannot be seen this far into the wing of the line.

The eruption shown in Figure 6 is also of the type described by Tang (1986) in which only the upper part of a filament actually erupts into the corona. Upper and lower segments are seen at $00: 15$ and $00: 22$. The upper part of the erupting filament is last 


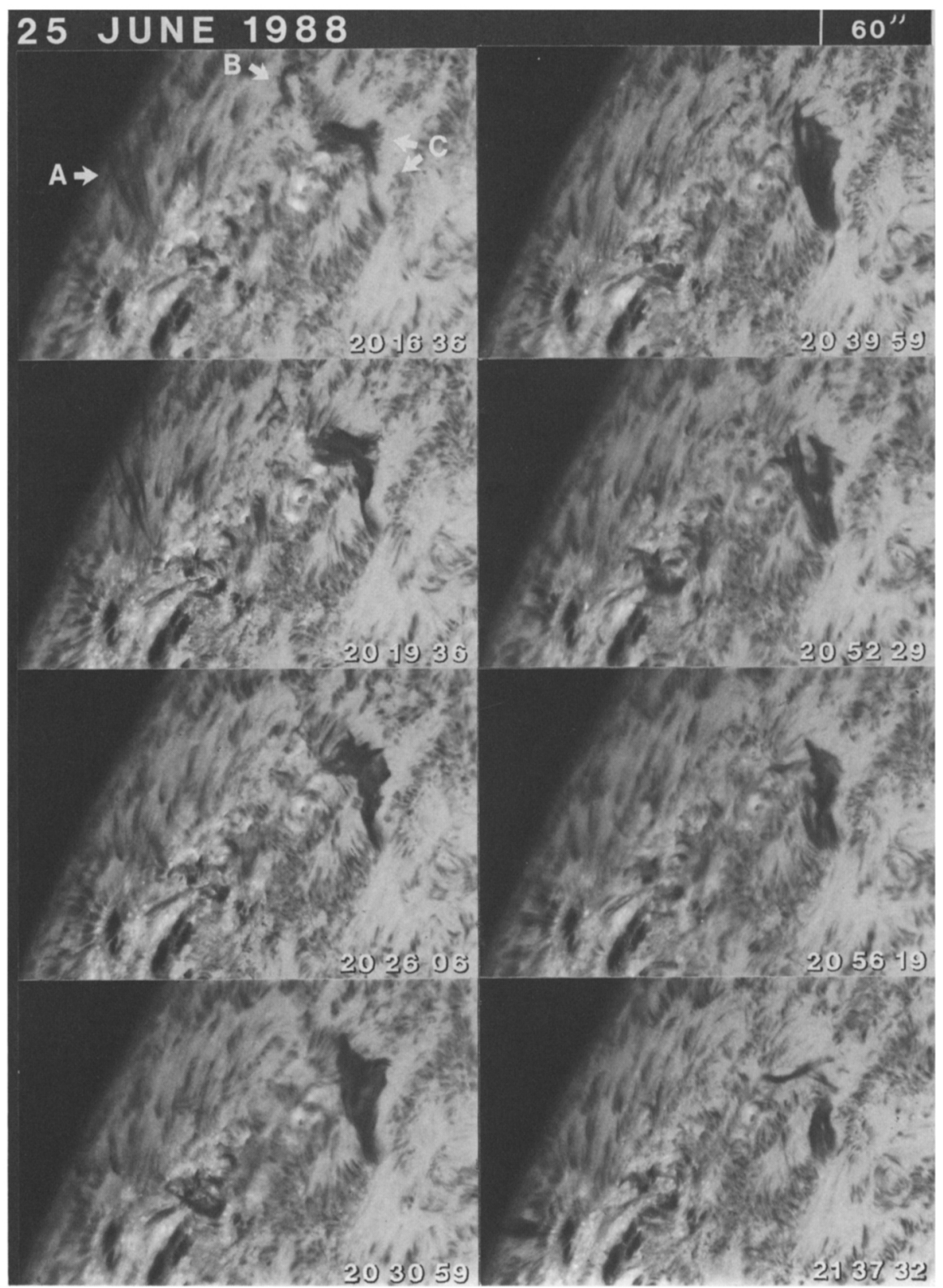

Fig. 7d. The activation of filament $C$ and the ending of the eruptions of filaments $A$ and $B$ are shown in these images at $\mathrm{H} \alpha-0.6 \AA$ in continuation of the time series in Figure 7(c).

seen above and to the right of the flare in the frame at $00: 32$. The lower part is activated and becomes the surge $(S)$ seen at $00: 32,00: 38$ and $00: 48$ in the lower part of each frame. Mass flows from the filament channel away from the flare. Around $00: 48$, the flow reverses and this mass then contributes to the formation of a new filament at the site of the eruption and activation. 
Surge-like activations within filaments occur frequently even in filaments which are not ready to erupt. Surge-like activations are most commonly triggered by small flares that occur near one end of a filament. A mass ejection is then observed to propagate along the filament. Such surge-like activations, triggered by small flares, were observed in the filament illustrated in Figure 6 several hours before the major flare that accompanied the eruption of the filament. In such a case, the mass flow typically runs along the filament first away from the flare site and then in the reverse direction. In addition, there can be highly turbulent flow patterns as were observed in this same filament at the Ottawa River Solar Observatory (V. Gaizauskas, personal communication).

Figures $7(\mathrm{a}-\mathrm{d})$ are an unusual series of images which depict, in a single field of view on 25 June, 1988, two erupting filaments with flares and the activation of a third filament from a mass ejecta associated with one of the flares. The filaments are identified as $A$, $B$, and $C$ in the upper frames of Figures $7(\mathrm{a}-\mathrm{d})$. Different aspects of preflare activation and eruption are seen in filaments $A(19: 43-23: 10)$ and $B(19: 43-23: 11)$. The activation due to impact of a mass ejecta takes place in filament $C(20: 16-21: 37)$ during the flare with the eruption of filament $B$. The culmination of these events is shown in images at the center of the $\mathrm{H} \alpha$ line in Figure 7(b). Figures 7(c) and 7(d) show the same sequence of events at $\mathrm{H} \alpha-0.6 \AA$.

Noteworthy features in this series of events are itemized below according to the nature of the activation.

\subsubsection{Preflare Activation and Flare-Related Eruption}

(a) The flare $(20: 10-20: 15$ Figure 7(a)) with filament eruption $A$ is an example in which some of the chromospheric and coronal parts of the flare are difficult to distinguish from each other. The starting time of the chromospheric ribbons below the ascending filament is uncertain (between 19:57 and 20:10) but the slow ascent of filament $A$ before its complete disruption is clearly revealed from $19: 43$ until $23: 10$. Its position at various times is confirmed by the blue wing observations in Figure 7(c). In the original timelapse film from which these images were selected, mass from the southern (upper) end of the filament appears to be ejected over the limb while down flow is seen in the northern leg of the filament until approximately $20: 33$. The rapid ejection of mass over the limb has in the past been called a 'spray'. Tandberg-Hanssen, Martin, and Hansen (1980) conclude that most 'sprays' have their origin in erupting prominences.

(b) The slow preflare ascent of filament $B$ is not readily seen in Figure 7(a) because of the orientation of the filament to our line-of-sight. However, the filament does darken and exhibit minor structural changes characteristic of the preflare ascending stage. The outward motion is confirmed in the corresponding blue wing $(-0.6 \AA)$ images in Figure 7(c) which show increased absorption from 20:07 until 20:16 and then the sudden disappearance of the filament between $20: 16$ and $20: 19$. It is common that the slow preflare ascent is not readily detectable in $\mathrm{H} \alpha$ filtergram images at line center except for events close to the limb. In many cases, however, the filaments observed at line center become noticeably darker during the hour prior to the flare. This is indirect evidence of either Doppler shift or spectral broadening of the filament, or both, during its preflare phase. 
(c) Filament $B$ changes from absorption feature to an emission feature in the images at $\mathrm{H} \alpha$ line center only during its expulsion phase (Figures $7(\mathrm{a})$ and 7 (b) at 20:15, 20:18 and $20: 20$ ). The transition to emission is possible evidence of heating.

(d) The activation of filament $C$ begins abruptly as an ejection of mass that hits the filament broadside at 20:15 (Figure $7(\mathrm{~A})$ ). The activation of this filament results in highly turbulent motion (Figures 7 (b) and 7(d)). In contrast, some activations via impact result in the organized bodily upward and downward motion of the whole filament (Ramsey and Smith, 1966; Harvey, Martin, and Riddle, 1974). This raises the question of whether activations have a different character depending upon whether the activation is initiated by mass ejecta, apparent in this case in Figure 7, or by a flare-related shock wave.

(e) The activation of filament $C$ is accompanied by faint emission (Figure 7(b)); such emission is not common for this type of activation.

\subsection{EXPANDING CORONAL FEATURES}

Observations of the coronal continuum above the limb from the coronagraph/ polarimeter experiments on both the Skylab and Solar Maximum Mission satellites show that most, if not all, erupting filaments are accompanied by a phenomenon now known as a 'Coronal Mass Ejection' (CME). A coronal mass ejection is an outwardly expanding volume of the corona that occupies a large amount of space above and around an erupting filament. CMEs are not discussed here in detail because recent reviews and general discussions of this phenomenon are given in Wagner (1984), Jackson (1985), Zirker (1985), and Hildner et al. (1986).

An outwardly expanding phenomenon, possibly related to CMEs, that occurs during the rise of a few impulsive flares, is the propagation of a broad front of diffuse emission away from the core of the flare. The diffuse emission may take either the form of an arc propagating away from a flare or of a brief emission halo around the core of a developing flare. Although these phenomena were first presented and discussed by Smith and Harvey (1971) along with evidence of flare-associated shock waves inferred from the oscillatory motions of filaments and chromospheric fibrils, these authors already gave reasons to doubt that the diffuse emission was an effect of the flare shock wave. They instead suggested that it represented another form of flare-associated mass motion. In 1980, at the Skylab Flare Workshop, I first proposed the hypothesis that the diffuse emission seen in filtergrams in Ca II and discussed in van Hoven et al. (1980) and Webb et al. (1980) could be a manifestation of a 'coronal transient', the phenomenon now known as a 'Coronal Mass Ejection' (CME), seen in projection against the disk. This idea has never been proven or disproven and no observations have yet been obtained (to my knowledge) in which this phenomenon has been observed concurrently with a CME. A primary problem in obtaining concurrent observations is that the diffuse emission is best seen with flares observed in the middle of the disk while the CME observations favor events near the limb.

Although the diffuse emission is often referred to as a 'Moreton wave', 'blast wave', or just 'flare wave', it is more likely that the real wave is invisible and that the emission is part of a mass ejection that possibly drives a shock wave through the corona. The strongest evidence of the existence of such shock waves in $\mathrm{H} \alpha$ images are the relatively 
rare observations of successive upward and downward motions of the chromospheric structures in an arc-shaped pattern that rapidly propagates away from a flare during its explosive rise to maximum (Moreton 1960, 1964, Moreton and Ramsey, 1960). This phenomenon, which we will call the 'chromospheric flare wave effect' has only been observed with a small percentage of very impulsive bright flares (Smith and Harvey, 1971). Downward and upward oscillations of distant filaments during flares is also good evidence of the existence of true shock waves from bright flares (Dodson, 1949; Ramsey and Smith, 1966; Harvey, Martin, and Riddle, 1974). It is less certain whether the flare shock wave can trigger point-like brightenings of the chromosphere outside of the flare (Smith and Harvey, 1971) because such brightenings could also be initiated by particles travelling along field lines directly from the associated flare to distant parts of the chromsophere as mentioned in Martin and Harvey (1972) and discussed in Sections 2.2 and 3.2 .

\section{Epilogue}

Solar flares represent the sudden release of energy in a localized parts of the solar atmosphere where oppositely directed magnetic fields are present. This release of energy results in various forms of mass motion whose geometry and velocity depend on the presence and varying configurations of the magnetic field. Some mass motions in the corona, such as those of erupting filaments, coronal mass ejections, and other expanding features, give evidence that the magnetic field is changing in the volume of space within and around the flare while others such as surges and flaring arches suggest that mass in these components is guided along a relatively static magnetic field configuration. Still other mass motions, in the chromospheric flare ribbons and adjacent chromosphere, are most likely the result of the impact of high speed particles and/or shock waves. Studies of these many forms of mass motion provide a means of deducing information about the changes in the magnetic field in the corona which cannot be directly detected in our present day magnetograms of the photosphere and chromosphere. The observed mass motions and related magnetic fields also provide constraints on the possible processes of energy release that take place during solar flares.

For a more comprehensive understanding of solar flares, it is hoped that this limited review of flare-related mass motions in the optical part of the spectrum will be followed by more comprehensive reviews of the different types of mass motions that are observed in flare components throughout the electromagnetic spectrum.

\section{Acknowledgements}

This review was accomplished under the sponsorship of the Air Force Office of Scientific Research under contract AFOSR-87-0023. The acquisition of new observational material presented herein was possible through support of the Big Bear Solar Observatory by NASA grant NGL05002034 and NSF grant ATM-8513577.

\section{References}

Canfield, R. C., Cheng, C. C., Dere, K. P., Dulk, G. A., and Schoolman, S. A.: 1980, in P. A. Sturrock (ed.), Solar Flares, A Monograph from Skylab Solar Workshop II, Appendix A, pp. 451-469.

Dodson, H. W.: 1949, Astrophys. J. 110, 382. 
Doschek, G. A. and 13 co-authors: 1986, in M. Kundu and B. Woodgate (eds.), Energetic Phenomena on the Sun, The Solar Maximum Mission Flare Workshop, NASA Conference Publ. 2439, pp. 4-1 to 4-42.

Ellison, M. A.: 1952, Publ. Rov. Obs. Edinburgh 1, 75.

Gaizauskas, V.: 1989, Solar Phvs. 121, 135 (this issue).

Harvey, K. L., Martin, S. F., and Riddle, A. C.: 1974, Solar Phys. 36, 151.

Hildner, E. and 20 co-authors: 1986, Energetic Phenomena on the Sun, The Solar Maximum Mission Flare Workshop Proceedings, NASA Conference Publ. 2439, Chapter 6.

Ichimoto, K. and Kurokawa, H.: 1984, Solar Phys. 93, 105.

Jackson, B. V.: 1985, Solar Phys. 100, 563.

Martin, S. F.: 1979, Solar Phys. 64, 165.

Martin, S. F.: 1980, Solar Phys. 68, 217.

Martin, S. F. and Harvey, K. L.: 1973, in A. J. Hundausen and Gordon Newkirk, Jr. (eds.), Flare-Produced Shock Waves in the Corona and in Interplanetary Space, Report on Conference held in Boulder, Colorado, 11-14 September 1972, High Altitude Observatory, National Center for Atmospheric Research.

Martin, S. F. and Ramsey, H. E.: 1972, in P. McIntosh and M. Dryer (eds.), Solar Activity, Observations and Predictions, MIT, Cambridge, p. 371.

Martin, S. F. and Švestka, Z.: 1988, Solar Phys. 116, 91.

Moore, R. L. and 16 co-authors: 1980, in P. A. Sturrock (ed.), Solar Flares, A Monograph from Skylab Solar Workshop II, pp. 341-409.

Moreton, G. E.: 1960, Astrophys. J. 65, 494.

Moreton, G. E.: 1964, Astron. J. 69, 145.

Moreton, G. E. and Ramsey, H. E.: 1960, Publ. Astron. Soc. Pacific 72, 357.

Mouradian, Z. and Soru-Escaut, I.: 1989, in B. M. Haisch and M. Rodono (eds.), IAU Colloq. 104, Solar and Stellar Flares, Poster Papers, Publ. Catania Astrophys. Obs., Special Volume, p. 227.

Mouradian, Z., Martres, M. J., and Soru-Escaut, L.: 1983, Solar Phys. 87, 309.

Ramsey, H. E. and Smith, S. F.: 1966, Astron. J. 71, 197.

Rust, D. M. and Bar, V.: 1973, Solar Phys. 33, 445.

Rust, D. M. and Webb, D. F.: 1977, Solar Phys, 54, 403.

Schadee, A. and Martin, S. F.: 1986, in D. Neidig (ed.), The Lower Atmosphere of Solar Flares, National Solar Observatory, Sacramento Peak Observatory, Sunspot, New Mexico, U.S.A., p. 360.

Schmahl, E. J., Webb, D. F., Woodgate, B., Waggett, P., Bentley, R., Hurford, G., Schadee, A., Schrijver, J., Harrison, R., and Martens, P.: 1986, Energetic Phenomena on the Sun, NASA Conference Publ. 2439, p. 1-48 to p. 1-72.

Schmieder, B., Simnett, G. M., Tandberg-Hanssen, E., and Mein, P.: 1988, Astron. Astrophys. (in press).

Schmieder, B., Forbes, T. G., Malherbe, J. M., and Machado, M. E.: 1987, Astrophys. J. 317, 956.

Schoolman, S. A. and Ganz, E. D.: 1981, Solar Phys. 70, 363.

Smith, S. F. and Harvey, K. L.: 1971, in Macris (ed.), Physics of the Solar Corona, pp. 156-167.

Smith, S. F. and Ramsey, H. E.: 1964, Z. Astrophys. 60, 1.

Sturrock, P. A.: 1980, in P. A. Sturrock (ed.), Solar Flares, p. 1-14.

Švestka, Z.: 1976, Solar Flares, D. Reidel Publ. Co., Dordrecht, Holland, pp. 76-80.

Švestka, Z., Kopecký, M., and Blaha, M.: 1962, Bull. Astron. Inst. Czech. 13, 37.

Švestka, Z., Martin, S. F., and Kopp, R. A.: 1980, in M. Dryer and E. Tandberg-Hanssen, Solar and Interplanetary Dynamics, p. 217.

Švestka, Z., Fárník, F., Fontenla, J., and Martin, S. F.: 1989, Solar Phy's. (in press).

Tandberg-Hanssen, E., Martin, S. F., and Hansen, R. T.: 1980, Solar Phys. 65, 357.

Tang, F.: 1983, Solar Phys. 83, 15.

Tang, F.: 1986, Solar Phys. 105, 399.

Tang, F. and Moore, R. L.: 1982, Solar Phys. 77, 263.

Teske, R.: 1962, Astrophys. J. 136, 534.

van Hoven, G. and 18 co-authors: 1980 , in P. A. Sturrock (ed.), Solar Flares, A Monograph from Skylab Workshop II, pp. 46-48.

Wagner, W. J.: 1984, Ann. Rev. Astron. Astrophys. 22, 267.

Waldmeier, M.: 1941, Ergebnisse und Probleme der Sonnenforschung, p. 197.

Webb, D. F., Cheng, C. C., Dulk, G. A., Edberg, S. J., Martin, S. F., McKenna-Lawlor, S., McLean, D. J.: 1980, in P. A. Sturrock, Solar Flares, A Monograph from Skylab Workshop II, Appendix B, pp. 471-499.

Wu, S. T. and 19 co-authors: 1986, Energetic Phenomena on the Sun, NASA Conference Publ. 2439, pp. 5-57 to $5-60$.

Zirin, H. and Tanaka, K.: 1973, Solar Phys. 32, 202.

Zirker, J. B.: Solar Phys. 100, 281. 